\title{
Psichiatria, architettura e sacro nella Vienna d'inizio secolo: alcune note su St. Leopold am Steinhof nel suo centenario
}

\author{
Con sei tavole
}

Il presente articolo sulla chiesa di St. Leopold am Steinhof (Fig. 1) intende correlare, attraverso la collaborazione di studiosi di diversa estrazione, i vicini ambiti delle scienze mediche e umane per una riflessione su psichiatria, architettura e sacro nella Vienna d'inizio secolo. Questo breve saggio è debitore di vari contributi critici riguardanti il clima culturale viennese dell'epoca, ma intende più specificatamente caratterizzarsi per il focus sulla considerazione della dimensione spirituale nella psichiatria dei primi del Novecento e quindi proprio sull'edificio sacro wagneriano, andando a integrare la scarsa bibliografia in lingua italiana dedicata specificatamente alla suddetta chiesa, della quale nel 2007 ricorreva il centenario del completamento della costruzione.

\section{LA PSICHIATRIA VIENNESE DI FRONTE AD ARTE E RELIGIONE}

Per comprendere in quale contesto sia stata commissionata l'opera di Wagner, è sicuramente importante esplorare il retroterra culturale della psichiatria viennese all'inizio del secolo scorso.

Da una parte alcuni professionisti lavoravano in grandi strutture di tipo manicomiale. Erano istituzioni estreme, che costituivano una sorta di enclave, nei territori di competenza, con una scarsissima permeabilità tra il mondo esterno, dei sani, e quello dei malati. Frequentemente si trattava di strutture mastodontiche, complessi con molti edifici e centinaia di degenti che spesso si trovarono lì confinati a trascorrere gran parte della loro vita. Il compito svolto dai medici in questo caso fu più il contenimento sociale della scomoda alterità rappresentata dal malato psichico, rispetto all'istanza della cura e della riabilitazione.

In tali strutture esisteva tuttavia per gli scienziati la possibilità di osservare la storia naturale delle malattie, il loro decorso naif, scevro da ogni intervento terapeutico. Dalla messe di osservazioni scaturisce nell'opera di Emil Kraepelin una minuziosa classificazione delle patologie, secondo una tassonomia di impronta quasi naturalistica. Alla concettualizzazione di psichiatri come Kraepelin e Bleuler si deve il filone medico della psichiatria, che riconosce in tali autori i propri padri fondatori. Questa corrente si limita ad un'osservazione ateoretica delle alterazioni mentali, comunque fenomeno incomprensibile ed incomunicabile. Nella cultura tedesca un altro filone stava però prendendo campo: Karl Jaspers nella sua Allgemeine Psychopathologie ${ }^{1}$ cominciò ad ipotizzare che almeno alcuni dei fenomeni psicopatologici fossero comprensibili ed interpretabili. Jaspers, tra l'altro, dedicò una delle sue opere principali ${ }^{2}$ al rapporto tra arte, genio creativo e malattia mentale. In tale libro, noto in Italia col titolo Genio e follia, Jaspers analizza la vita del drammaturgo, scrittore e pittore Strindberg e dipinge come un unico itinerario umano e culturale il percorso creativo e lo scivolare nel delirio dell'artista svedese. Soprattutto la nascita della psicanalisi porterà a compimento il filone della psicopatologia interpretativa, ritenendo ogni fenomeno comprensibile per lo psichiatra che conosca la storia della vita del suo paziente. Gli psicanalisti cominciarono inoltre ad occuparsi di un tipo diverso di malati, che non richiedevano di essere internati, ma si recavano allo studio dello psicoterapeuta per affrontare le proprie nevrosi o problemi esistenziali.

Rispetto ai 'grandi psicotici', con questo tipo di pazienti era possibile un confronto più serrato dal punto di vista dialettico; molti di loro provenivano dall'alta borghesia europea ed alcuni erano artisti o creativi.

\footnotetext{
*Dipartimento di Psichiatria, Neurobiologia, Farmacologia e Biotecnologie, Università di Pisa.

**Dipartimento di Storia delle Arti, Università di Pisa.

${ }^{1}$ K. JASPERS, Allgemeine Psychopathologie. Berlin 1913.

${ }^{2}$ ID., Strindberg und van Gogh: Versuch einer pathographischen Analyse unter vergleichender Heranziehung von Swedenborg und Hölderlin. Bern 1922.
} 
Proprio dall'incontro con uno di questi pazienti nel suo studio viennese, paziente che ha "la fisionomia di Leonardo da Vinci", scaturisce l'interesse di Freud per la vita del pittore italiano che lo porterà a scrivere una delle sue prime psicobiografie ${ }^{3}$.

Vienna, all'inizio del XX secolo, rappresentava un luogo d'incontro di idee e uomini provenienti da diversi percorsi intellettuali, $\mathrm{i}$ quali fornirono un contributo di inusitata ricchezza alla psichiatria come branca nascente della medicina.

Richard von Krafft-Ebing muore nel 1902, dopo aver trascorso gli ultimi anni della sua vita professionale proprio nella capitale dell'Impero asburgico, come professore nella locale università. Figura complicata e perfino controversa, Krafft-Ebing ebbe una vasta produzione scientifica, ma la sua notorietà gli derivò soprattutto dalla sua Psychopathia sexualis ${ }^{4}$, che analizza e descrive centinaia di casi clinici di disturbi e perversioni sessuali giunti all'attenzione dello psichiatra. Per alcuni anni le sue specifiche competenze resero Krafft-Ebing il riferimento naturale per gli aspetti forensi della psicopatologia e la sua collaborazione con le istituzioni fu intensa.

In quel periodo egli avanzò una teoria che affrontava in modo nuovo alcune tematiche religiose, fornendone una spiegazione in chiave sessuologica. In particolare, però, l'accostamento del desiderio di santità e del martirio, narrati in alcune agiografie, al masochismo, perversione da lui per primo analiticamente descritta e classificata, gli attirò le inimicizie della gerarchia ecclesiastica austriaca, isolandolo sul piano politico-istituzionale.

Un rapporto contrastato con l'autorità religiosa segna tutto il percorso degli psichiatri in quel periodo. Nello stesso anno in cui muore Krafft-Ebing, Sigmund Freud ottiene la cattedra di professore straordinario all'Università di Vienna. Ormai il paradigma psicoanalitico sta diventando un tema di confronto per gli psichiatri ed il suo impatto nella cultura in genere comincia a caratterizzarsi per quello che sarà nella metà successiva del secolo, quando influenzerà, in un ricco rapporto biunivoco, arte e letteratura. Se l'ambiente accademico è generalmente intrigato dalla teoria freudiana, diverso è il rapporto con la Chiesa che rimane sospettosa verso la centralità degli aspetti sessuali e la visione materialistica della psiche che caratterizza Freud ed epigoni. Questa posizione cessa di essere prevalente già nel 1910, quando viene eletto come presidente della società degli psicoanalisti Carl Gustav Jung, che invece incentrerà molto della sua teoria sulla religiosità come vis naturalis che conduce l'uomo ancora più degli istinti sessuali. Tuttavia, questo porta alla rottura proprio con il gruppo viennese, che rimane in linea con l'ortodossia freudiana.

L'ineguagliabile fermento di riflessioni su questi temi che si vive in quegli anni a Vienna, riesce a costruire la nuova immagine dello psichiatra che sarà il prototipo del 'medico dell'anima' fino ad oggi. Una figura di intellettuale di formazione scientifica, ma non estraneo a quella cultura umanistica, artistica e filosofico-religiosa che risultano imprescindibili per la comprensione dell' animo umano.

Un recupero della tradizione umanistica, proprio quando la psichiatria tende a rendersi branca autonoma nell'ambito di una medicina sempre più scienza biologica, conferirà a questa materia il ruolo di ponte tra discipline ed interessi apparentemente molto distanti.

Interprete emblematico di tale esperienza nella Vienna a cavallo tra fine Ottocento e inizio Novecento fu Arthur Schnitzler, medico e scrittore. Nella sua vita, ancor prima che nella sua opera, si fondono l'istanza scientifica e quella artistica. Il lavoro di composizione di queste realtà per raggiungere la completezza dell'individuo lo impegnerà per tutta la vita. Nel suo romanzo breve Traumnovelle ${ }^{5}$, incentrato sul mondo onirico dei protagonisti, vengono affrontati alcuni punti cardine del lavoro psicoanalitico come ricerca di una sintesi tra il mondo diurno, cosciente e razionale, e quello notturno, atavico, simbolico, e talora perturbante, del sogno o dell' arte.

In questo ambiente culturale comincia a essere comprensibile la ricerca di sinergie e la progressiva apertura del mondo dei 'malati' a quello dei 'sanil'e la possibilità per i pazienti di riappropriarsi di alcuni aspetti della vita intellettuale e spirituale fruendo dell' arte e della religiosità. Da tali presupposti consegue anche la ricerca di una diversa spazialità ospedaliera che rappresenti una triplice riunificazione: tra la società e la malattia mentale, prima nettamente divise, tra l'intervento del medico e la soggettività dell'uomo sofferente, ed infine tra le varie componenti della complessa natura dell'animo umano.

\footnotetext{
${ }^{3}$ S. FREUD, Eine Kindheitserinnerung des Leonardo da Vinci. Leipzig und Wien ${ }^{1} 1910,{ }^{2} 1919$.

${ }^{4}$ R. von KraffT-Ebing, Psychopathia sexualis: eine klinisch-forensische Studie. Stuttgart 1886.

5 A. SCHNITZLER, Traumnovelle. Berlin 1926.
} 


\section{IL COMPLESSO DELLO STEINHOF NELL'OPERA DI OTTO WAGNER}

Otto Wagner selezionò e raccolse in quattro volumi i disegni più significativi della sua produzione, dandogli il titolo Einige Skizzen, Projekte und ausgeführte Bauwerke. Il terzo volume, pubblicato nel 1906 dall'editore Anton Schroll di Vienna, conteneva anche alcuni schizzi e progetti per la chiesa di St. Leopold am Steinhof. Tale edificio gli fu commissionato dal Landtag, il parlamento provinciale della Bassa Austria, il 12 novembre 1903, dopo una vivace discussione della proposta di Wagner, di quella del suo allievo Leopold Bauer e di una terza del Landesbauamt, unici candidati di un concorso a inviti bandito nel 1902. Nella medesima seduta fu respinto il piano generale dell'intero complesso psichiatrico presentato dal Landesausschuss, mentre l'esplicita autocandidatura dello stesso Wagner con una diversa planimetria, avvenuta in una posizione d'onore come consulente artistico, gli valse l'approvazione del parlamento provinciale relativamente ai principi ispiratori della disposizione degli edifici, e fu poi proprio il suo progetto ad essere seguito ed eseguito, seppure con alcune varianti. A tale estensione dei compiti di Wagner si deve anche un famoso disegno dell' asse centrale del complesso per mostrare la chiesa in rapporto al circondario, disegno nel quale appaiono, da un lato, evidenti suggestioni barocche, per l'articolazione curvilinea di alcuni percorsi nei giardini, e richiami alla collocazione della gloriette di Schönbrunn proposta da Hetzendorf von Hohenberg, dall'altro lato, citazioni dello stesso Wagner nei progetti "Artibus" o per l'Accademia di belle arti di Vienna. Il piano generale dell'ospedale psichiatrico, per quanto richiedesse movimenti di terra dispendiosi per livellare i vari piani su cui Wagner disponeva gli edifici, evitava con una strutturazione compatta l'acquisto di nuovi terreni da parte dell'amministrazione regionale ${ }^{6}$ e mostrava un sapiente equilibrio tra la varietà delle singole costruzioni e la chiara razionalità dell'impianto ospedaliero per malati curabili e incurabili, della zona residenziale per i pazienti paganti, dei servizi e delle zone d'isolamento per i malati criminali.

Le caratteristiche dell'edificio religioso, la pianificazione del complesso e soprattutto le modalità con cui il grande architetto affrontò il tema della chiesa e dell'ospedale psichiatrico vanno ricercate nella storia recente del professore e progettista.

Wagner nel 1895 aveva esposto nel volume Moderne Architektur nuovi principi su cui fondare l'architettura contemporanea, rifiutando di applicarsi coi suoi allievi solo al consueto problema didattico delle forme e della costruzione, e perorando l'importanza della rispondenza alle esigenze dei tempi, in luogo dell'attenzione alle tradizionali codificazioni stilistiche, convinto che non sarebbe mancata agli architetti del XX secolo la possibilità di declinare la creatività in vari linguaggi, risultato anche dell'impiego dei nuovi materiali. La teoria anticipa nuove soluzioni più rapidamente che la pratica wagneriana, ma leggendo assieme Moderne Architektur e il progetto per il complesso dello Steinhof e la sua chiesa, si troveranno senz'altro interessanti punti di tangenza.

Wagner, nel suo scritto, affronta il ruolo dello stato nella protezione dell'architettura di qualità, auspicando sia l'affidamento della costruzione di edifici pubblici solo a professionisti scelti tramite concorsi, sia la promozione della formazione accademica. Tuttavia afferma che anch'essa è insufficiente se l'architetto è privo della necessaria sensibilità "ai bisogni dell'uomo"7 o si rifugia in un linguaggio incomprensibile al pubblico. Si comprenderà poi, al momento della progettazione dello Steinhof, quanto valore avesse di fronte alla città e al governo austriaco la figura di Wagner, tanto da selezionarlo vincitore del concorso a inviti per la chiesa e da accettarne una autocandidatura per la revisione del piano generale dell'ospedale psichiatrico. In un' annosa vicenda biografica di commissioni pubbliche affidate o progetti scartati, in un rapporto di stima o indifferenza della municipalità viennese alle proposte dell'architetto, l'affidamento di incarichi relativi all'importante realizzazione del complesso dello Steinhof segnò un momento di gloria per Wagner.

Mai come nell'ospedale e nella chiesa egli fu chiamato a dar prova di essere veramente attento ai bisogni umani, sia fisici che spirituali, assecondando le esigenze di funzionalità di tali volumi architettonici. Per le creazioni architettoniche contemporanee, Wagner invoca uno stile originale: "L'arte moderna deve offrire forme nuove che rappresentino la nostra epoca e il nostro modo di vivere" ${ }^{\text {e }}$ " $L$ ' arte e le sue forme devono

\footnotetext{
${ }^{6}$ Cfr. R. Trevisiol, Otto Wagner. Roma-Bari 1997, 164.

7 O. WAGNER, Architettura moderna ed altri scritti. Bologna 1980, 56.

${ }^{8}$ IBID., 56.
} 
assolutamente esprimere le tendenze ed i fenomeni della vita moderna"’9. Negli spazi dello Steinhof, per quanto si possano riscontrare per la chiesa suggestioni derivate dall'intera storia dell'architettura sacra, si segue il principio wagneriano del Nutzstil, dello stile dell'utilità, per cui "niente che non sia funzionale potrà mai essere bello",10.

La perfetta integrazione di architettura e arti decorative, l'opera d'arte curata nei dettagli secondo i modi della Secessione viennese, non lasciano comunque spazio al superfluo: dalla committenza si richiese del resto bellezza e praticità, elementi essenziali alla terapia stessa dei malati di mente. Un ambiente naturale e architettonico gradevole svolgeva, secondo i medici, un ruolo importante nella vita del paziente, mentre una disposizione economica e pragmatica degli ambienti e degli arredi soddisfaceva la richiesta della committenza di edificare il più grande e moderno ospedale psichiatrico d'Europa razionalizzando le comunque ingenti spese.

In Moderne Architektur Wagner sostiene che "ogni nuova creazione, se vuole essere veramente moderna, deve corrispondere alle esigenze del nostro tempo e ai nuovi materiali, deve esprimere al meglio la nostra mentalità democratica e responsabile, deve tener conto delle enormi conquiste tecniche ed economiche e dello spirito pratico tipico dell'uomo moderno"11.

Di tale mentalità e delle conquiste mediche e scientifiche la committenza dello Steinhof voleva dar mostra nel nuovo ospedale psichiatrico, dove i malati non sarebbero stati più come in passato considerati sempre incurabili, pericolosi, asociali, disumani, diversi e quindi esclusivamente segregati o gestiti come in un carcere. Il sogno ottimista di una nuova comunità di recupero e di una cittadella ideale, o il disegno di un complesso razionale e ricco di risorse per i degenti che si sperava contribuisse a dare ordine alla follia ivi contenuta, si configuravano comunque in uno spazio secluso, lontano dal centro di Vienna, quanto bastava per poter allontanare dalla vista una realtà sempre temuta o per darle il pittoresco aspetto di "città bianca"12 adagiata sul colle (Fig. 2), della quale emergevano soprattutto la monumentale chiesa o la direzione, mentre reparti e zone di riabilitazione, mura divisorie e celle di contenimento restavano discretamente occultate dalla vegetazione.

Proprio il rapporto del complesso dello Steinhof con Vienna era un fondamentale problema urbanistico. Wagner aveva avuto l'incarico di stendere il piano regolatore generale della città, si era occupato dei trasporti metropolitani e si avviava a riflettere ancora sui nuovi distretti di una città in espansione, perciò quando presentò il suo progetto per lo Steinhof lo articolò in modo che strade, sentieri, giardini interni seguissero una griglia collegabile a viali e spazi verdi all'esterno.

Nel libro Moderne Architektur aveva già invitato il progettista a pensare con attenzione all'effetto d'insieme della sua opera e agli effetti prospettici o visivi, tenendo conto del genius loci e "del luogo, del tempo, della moda" 13 , nonché insistendo sulla bellezza della simmetria. Proprio la distribuzione delle sezioni maschile e femminile dell'ospedale psichiatrico in base ad un asse di simmetria costituito dagli spazi accessibili ai visitatori esterni, come gli edifici amministrativi o il teatro e culminante con St. Leopold che va a costituire il punto focale dell'intero complesso, dimostra la coerenza dell'architetto con la sua teoria e va ad assecondare: l'esigenza gestionale di una terra di nessuno tra i due generi di pazienti ${ }^{14}$, l'inclinazione del terreno che prevede un'ascesa dal cancello d'ingresso dell'ospedale (Fig. 3) alla chiesa, e forse anche la persistenza nella mentalità collettiva di una fiducia nel "potere dello spirito sull'irrazionalità della malattia e sulla semplice gestione razionale della follia" $"$, affidando alla divinità un ruolo preminente nella tutela ultima delle sorti del malato, benché con la medicina moderna si potessero fornire nuovi ausili alla sua salute mentale.

\footnotetext{
${ }^{9}$ IBID., 62.

${ }^{10}$ IBID., 67.

${ }^{11}$ IBID., 65.

12 Secondo la definizione che ne dette Ludwig Hevesi vedendola dal treno. Cfr. L. HeveSI, Otto Wagners moderne Kirche, in: IDEM, Altkunst-Neukunst. Wien 1894-1908. Wien 1909, 249.

13 WAGNER (come nota 7), 70.

${ }^{14}$ Fin dalla fine del XVIII secolo, quando si pensò ad un sistema di trattamento individualizzato dei pazienti, classificandone le diverse tipologie, la separazione spaziale l'una dall'altra sembrava essenziale al superiore senso di ordine e razionalità cui doveva informarsi l'ospedale psichiatrico. Tra le basilari distinzioni da mettere architettonicamente in atto ci fu quella tra uomini e donne, giustificata in testi specialistici per il timore di eventuali violenze sessuali e procreazioni all'interno del manicomio. Cfr. C.F.W. RoLLER, Die Irrenanstalt nach allen ihren Beziehungen. Karlsruhe 1831, 73.

15 P. HAiko-H. Leupold-Löwental-M. Reissberger, La ville blanche "au Steinhof" a Vienne: architecture et maladie mentale, in: Vienne 1880-1938. L’Apocalypse joyeuse, a cura di J. ClaIR. Paris 1986, 260.
} 


\section{LA CHIESA DI ST.LEOPOLD: L'ARCHITETTURA SACRA SECONDO OTTO WAGNER}

Il percorso compiuto da Otto Wagner nell'elaborazione di edifici sacri si apre con la sinagoga di Budapest, concepita attorno al 1870 in uno stile moresco basato su di un impianto ottagonale e sulla ripetizione di tale figura geometrica anche negli arredi e negli elementi minori. L'architetto si dedicherà poi a luoghi di culto cristiani, a partire nel 1879 dal progetto per una chiesa a Soborsin, in cui emerge la predilezione per le cupole, per poi proseguire nel 1890 col progetto per la parrocchiale di Esseg (Osijek, Croazia), fortemente caratterizzata dalla struttura in ferro a vista, con slanciati pilastri che dividono le tre navate. Wagner appronterà nel 1891 uno studio sul duomo di Berlino, per poi dedicarsi a una piccola opera viennese, la Johanneskapelle realizzata nel 1895 sul Gürtel in pianta centrale con cupola. Al 1898 risalgono sia il progetto di chiesa parrocchiale per il cimitero di Währing, ideata su pianta quadrata e culminante in una cupola emisferica, sia il progetto di ristrutturazione della chiesa dei Cappuccini e della cripta imperiale. L'anno seguente, il 1899, l'architetto espone nel Die Moderne im Kirchenbau le sue riflessioni grafiche sui modelli del passato e le sue recenti interpretazioni del tema, avviandosi a delineare la pianta centrale e la cupola come elementi paradigmatici per l'edificio cristiano. Nel 1902 Wagner partecipa a due concorsi di architettura sacra, quello per la cattedrale di Patrasso e quello per la chiesa di St. Leopold am Steinhof a Vienna. Il secondo lo vede vincitore e dopo una rielaborazione tra 1903 e 1904 dei disegni, implicante principalmente l'eliminazione della cripta e di una scalinata prevista dall'architetto ma non gradita dalla committenza, Wagner si troverà a dirigere i lavori di costruzione, compiuti tra 1905 e 1907 con l'assistenza di Marcel Kammerer e Otto Schönthal.

Questo sarà l'ultimo edificio religioso realizzato da Wagner, sebbene tra 1906 e 1907 abbia ancora affrontato a livello grafico il tema dell' architettura sacra, progettando varie tipologie di avveniristiche chiese provvisorie, componibili come prefabbricati grazie ai nuovi materiali da costruzione. L'ultima incursione progettuale in campo cristiano di Otto Wagner fu infine nel 1917 l'ideazione della cosiddetta 'chiesa dell'armistizio' o 'della pace', inizialmente denominata 'chiesa della vittoria' e prudentemente ribattezzata vedendo che le sorti della guerra mondiale stavano volgendo nettamente a sfavore dell'Austria-Ungheria. Wagner considerava la chiesa dell'armistizio l'edificio monumentale con cui passare alla storia, riponendo grande entusiasmo per questo complesso costituito da un recinto rettangolare, aperto su un lato verso $\mathrm{i}$ quartieri della nuova Vienna progettati dallo stesso Wagner, e da una chiesa a pianta centrale, con annesso campanile.

Di fatto è invece proprio la chiesa di St. Leopold a essere considerata dai contemporanei il primo vero edificio monumentale di Wagner, come Otto Schönthal scrive sul periodico Der Architekt nel $1908^{16}$. Proprio sulle colonne del medesimo periodico sono stati ospitati commenti e critiche all'opera di Wagner per tutta la durata della sua costruzione: già nel 1905 Joseph Lux nell' articolo Zum modernen Kirchenbau sottolineava ${ }^{17}$ che le componenti pratiche nel progetto di una chiesa moderna dovevano essere il riscaldamento, la luce, l'igiene, l'acustica e la visibilità dell'altare. St. Leopold sembrava essere la risposta. Ferdinand Feldegg, sempre su Der Architekt nel $1905^{18}$, accusa invece Wagner di addobbare di maschere l'edificio, senza che la tanto proclamata 'verità strutturale' possa esservi trovata, e insinua anche che St. Leopold abbia ben poco di spirituale, principalmente "la casa di Dio per Otto Wagner è il teatro di Dio: ognuno può ascoltare e vedere bene da ogni posto".

Se gli obiettivi dichiarati dal grande maestro furono la velocità di costruzione, il contenimento dei costi, l'igiene, la qualità acustica e visiva dell'interno, la funzionalità dell'edificio, essi vennero raggiunti con un'architettura di qualità pratica ed estetica, tentando in questa chiesa di incarnare il principio wagneriano della necessità come unica guida all'arte.

Wagner concepì uno spazio per 800 persone, col pavimento inclinato con $30 \mathrm{~cm}$ di dislivello verso l'altare per agevolare la congregazione a dirigere l'attenzione verso il sacerdote. I piccoli banchi per 400 posti erano progettati per permettere lo spostamento di pazienti che subissero improvvise crisi, una stanza di

\footnotetext{
${ }^{16}$ Cfr. O. SchÖNTHAL, Die Kirche Otto Wagners. Der Architekt 14 (1908), 1-6.

${ }^{17}$ Cfr. J. LuX, Zum modernen Kirchenbau. Der Architekt 11 (1905), 6.

18 Cfr. F. FelDEGG, Literatur. Ibid., 16.
} 
primo soccorso si trovava nella chiesa. Tutto fu programmato in vista di una buona luminosità: se non c'è finestra dietro l'altare (Fig. 4) perché Wagner pensava che potesse disturbare gli ammalati con l'illuminazione diretta, sono presenti le grandi vetrate sui lati, mentre delle feritoie nel tamburo della cupola dirigono la luce sulle vetrate del sottostante controsoffitto voltato, realizzato alle cosiddette piastre Rabitz chiare per riflettere la luce e utile a migliorare l'acustica. Il sacerdote ha diretto accesso al pulpito dalla sacrestia senza entrare nella navata, onde evitare di esporsi rischiosamente ai pazienti più agitati. Al servizio dell'igiene, Wagner scelse il bianco come colore predominante delle pareti e non creò angoli difficili da pulire. Un getto d'acqua benedetta al posto dell'acquasantiera a bacile serviva per prevenire la trasmissione di infezioni.

Ulteriori accorgimenti pratici furono una stanza per le confessioni dei deboli d'udito o dei pazienti più rumorosi, la riduzione al minimo di spigoli coi quali i malati si potessero ferire, la separazione del settore maschile e femminile dei seggi con l'interposizione delle panche per il personale in servizio nell'ospedale e i relativi accessi separati per sesso da porte diverse.

A contribuire alla realizzazione degli arredi interni Wagner chiamò vari artisti, in primis l'amico secessionista Koloman Moser, al quale furono affidate le grandi vetrate della chiesa, ma non, come avrebbe desiderato l'architetto, le pale d'altare musive. Nel 1905-06, infatti, il sacerdote Heinrich Swoboda, forte anche del giudizio negativo del famoso prelato Wilpert, specialista di arte cristiana, polemizzò sullo stile di Moser, giudicato troppo pagano e sensuale. I contributi musivi sopra l'altare maggiore furono eseguiti da Remigius Geyling, mentre gli altari laterali furono affidati a Rudolf Jettmar, e si giunse alla loro completezza solo nel 1913. Si ricordano tra i partecipanti all'impresa Paul Neumann, autore del pulpito, e Franz Josef Swoboda, che costruì l'organo. All'esterno, Richard Luksch si occupò delle statue dei santi Severino e Leopoldo (Fig. 5) per i due campanili, mentre Othmar Schimkowitz realizzò i quattro angeli sulla facciata principale, oltre quelli del baldacchino dell'altar maggiore.

Non potendo soffermarsi in questa sede sui vari artisti, avendo di mira soprattutto l'architetto e il suo rapporto col sacro nella chiesa dell'ospedale psichiatrico am Steinhof, sarà importante citare per esteso i pareri di due contemporanei. In occasione della dedicazione dell'altare, avvenuta il 26 luglio 1913, l'arcivescovo di Vienna Friedrich Piffl disse: "sono specialmente grato per l'opportunità di partecipare a questa cerimonia, perché mi ha dato la possibilità di rendermi conto personalmente di questo monumentale capolavoro d'architettura sacra, che ho sentito così frequentemente mettere in discussione da coloro che sono preparati a poterlo fare. Io posso solo dire una cosa: che io stesso, quando sono entrato nella chiesa, sono stato letteralmente sopraffatto dal carattere monumentale dell'edificio. Sentivo emergere la sensazione che 'questo è veramente quel posto sacro, del quale fu scritto, levati le scarpe, abbandona dietro di te tutti i pensieri mondani e volgiti a Lui per il quale questa casa di Dio fu creata!'. Signor Wagner, io rimarrò sempre un difensore di questa chiesa, che produce un effetto così monumentale, che dichiara di se stessa che è la casa di Dio" ${ }^{19,}$.

A tale appassionata difesa dell'opera di Wagner e all'apprezzamento di una maestosità che invitasse il fedele all'elevazione verso Dio nella preghiera, si contrappone l'osservazione di Hans Tietze, che nel 1922 scrisse che St. Leopold era "un edificio che adempie perfettamente a tutti i requisiti negativi; è igienico e organizzato chiaramente, pratico e festoso, ma gli manca l'atmosfera della devozione religiosa. È una hall festiva per una funzione liturgica, uno spazio cerimoniale per una religione razionalista, una specie di chiesa per i non ecclesiastici, molto caratteristica del pensiero del tempo della sua creazione, come maschera di un essere non toccato, come un pezzo pomposo di decorazione, che prova la vittoria della necessità artistica sulla percezione artistica ${ }^{20}$. Wagner in persona ammise nel suo diario che negli anni della costruzione di St. Leopold si era distaccato da ogni religione, non confidando più in un Dio che non gli si mostrava e interrogandosi sulla caducità dell'uomo, cui non ci si poteva contrapporre se non, da architetto, credendo almeno a un'eterna sopravvivenza delle sue opere. L'impegno profuso a creare St. Leopold fu per Wagner l'estrinsecazione di un desiderio di immortalità professionale, ma anche la concretizzazione in uno spazio della possibile alternativa artistica alla sua spiritualità inquieta e alla totale disillusione, il dar forma architettonica almeno all'evocazione di un senso di pace e di bellezza, ricercate anche dai pazienti dello Steinhof, seppur fosse perduta o a tratti incomprensibile la fede cristiana. Così in una Vienna di inizio secolo, al bivio tra tradizione e modernità, tra cattolicesimo e altre forme di pensiero, un professionista come Wagner infuse un senso spirituale al suo ultimo edificio sacro, leggibile a vari livelli, come la casa di Dio o

${ }_{20}$ Cit. in P. Vergo, Art in Vienna 1898-1918: Klimt, Kokoschka, Schiele and their contemporaries. London 1975, 109-110.

${ }^{20}$ H. TiETZE, Otto Wagner. Wien 1922, 11. 
quella di una possibile pragmatica riconciliazione col destino perituro e il dolore dell'uomo, attraverso la vitalità dell' arte e l'architettura.

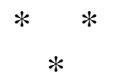

\section{CONCLUSIONI}

Dal punto di vista storico architettonico si deve rilevare che la tipologia di St. Leopold (Fig. 6), chiesa a pianta centrale, si ritrovò spesso nei lavori degli allievi di Wagner all'inizio del Novecento, i loro studi elaboravano il tema dell' antico heroon (recinto sacro per l'eroe) combinando il volume coperto a cupola con una torre campanaria. La Wagnerschule, attenendosi a questa tipologia, raccolse consensi e commissioni in tutta l'Europa centro-orientale ${ }^{21}$. Questi architetti furono anche frequentemente chiamati, sul modello di Wagner o Hoffmann, a affrontare il tema dell'ospedale o casa di cura in tutti i territori dell'Impero asburgico $^{22}$.

Per quanto riguarda la storia della medicina, il complesso dello Steinhof segnò effettivamente una pietra miliare, essendosi proposto quale "un manuale costruito della moderna psichiatria" ${ }^{23}$, tanto da divenire meta di viaggi di formazione per gli specialisti di vari paesi, interessati a conoscere dal vivo e scambiare opinioni con i colleghi di quello che effettivamente restò per lungo tempo la più grande e moderna struttura ospedaliera psichiatrica d'Europa.

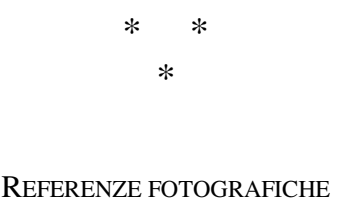

Fig. 1-6: Historisches Archiv des Otto Wagner-Spitals, Wien (foto di Paul-Johannes Keiblinger)

${ }^{21}$ Vedi A. MorAVÁNSZKY, Competing visions: aesthetic invention and social imagination in Central European architecture $1867-$ 1918. Cambridge (Mass.) 1998.

${ }^{22} \mathrm{Va}$, in questo contesto, ricordato l'ospedale psichiatrico a Kroměříž in Moravia, realizzato a partire dal 1905 da Hubert Gessner, allievo di Otto Wagner. Si tratta di un'opera architettonica che può definirsi un riflesso immediato del grande complesso viennese sia per la planimetria generale che per la chiesa dei Santi Cirillo e Metodio, molto simile a St. Leopold am Steinhof. Cfr. J. VyBíRAL, Der Traum von der Vernunft unter der gelben Kuppel: die Architektur von Hubert Gessner in Kremsier. Umění/Art 47/1-2 (1999), 45-58.

${ }^{23}$ L. TopP, Otto Wagner and the Steinhof Psychiatric Hospital: architecture as misunderstanding. ArtBull 87/1 (2005), 138. 